\title{
On polynomial symbols for subdivision schemes
}

Given a dilation matrix $A: \mathbb{Z}^{d} \rightarrow \mathbb{Z}^{d}$, and $G$ a complete set of coset representatives of $2 \pi\left(A^{-\top} \mathbb{Z}^{d} / \mathbb{Z}^{d}\right)$, we consider polynomial solutions $M$ to the equation $\sum_{g \in G} M(\xi+g)=1$ with the constraints that $M \geq 0$ and $M(0)=1$. We prove that the full class of such functions can be generated using polynomial convolution kernels. Trigonometric polynomials of this type play an important role as symbols for interpolatory subdivision schemes. For isotropic dilation matrices, we use the method introduced to construct symbols for interpolatory subdivision schemes satisfying Strang-Fix conditions of arbitrary order. 